\title{
Biogeography-inspired multiobjective optimization for helping MEMS synthesis
}

\author{
Paolo di Barba ${ }^{1}$, Maria Evelina Mognaschi ${ }^{1}$, PaOlo Venini ${ }^{2}$, Slavomir Wiak ${ }^{3}$ \\ ${ }^{1}$ Department of Electrical, Computer and Biomedical Engineering, University of Pavia \\ Via Ferrata 5, 27100 Pavia, Italy \\ e-mail:eve.mognaschi@unipv.it \\ ${ }^{2}$ Department of Civil Engineering and Architecture, University of Pavia \\ Via Ferrata 3, 27100 Pavia, Italy \\ ${ }^{3}$ Institute of Mechatronics and Information Systems, Lódz University of Technology \\ Stefanowskiego 18/22 Str., 90-924 Lódz, Poland
}

(Received: 03.02.2017, revised: 20.03.2017)

\begin{abstract}
The aim of the paper is to assess the applicability of a multi-objective biogeography-based optimisation algorithm in MEMS synthesis. In order to test the performances of the proposed method in this research field, the optimal shape design of an electrostatic micromotor, and two different electro-thermo-elastic microactuators are considered as the case studies.
\end{abstract}

Key words: biogeography-inspired optimisation, finite-element method, MEMS, multiphysics analysis, Pareto optimality

\section{Introduction}

The automated optimal design of devices and components in electricity and magnetism is usually performed by means of evolutionary or genetic algorithms [1-3], among which, NonDominated Sorting Genetic Algorithm (NSGA-II) is one of the most popular and well-assessed [4]. Recently, optimisation algorithms inspired by a migration paradigm have been developed: they might be considered as cooperative algorithms, while the evolutionary and genetic ones are rather competitive algorithms. In fact, in cooperative algorithms there is an exchange of information between individuals which autonomously evolve. The cooperative algorithms based on the migration paradigm mimick an exchange of species between small islands $[5,6]$. The underlying model is the phenomenon of natural population evolving without exchange with the external environment, such as those that might occur within small islands with limited migration. The assumption of small island is important in order to exclude surface-related local perturbations, like e.g. climate variations, that might make a subregion most preferred to other subregions of the same island. 
As far as the design of integrated devices and systems is concerned, the impressive advance of technology at both the micrometer and nanometer scales requires the development of powerful and flexible modelling tools to help the designer [7-9]. For instance, in [10] a topology optimisation method to design a piezoelectrically-driven microgripper is proposed: four design criteria are considered, and the two-dimensional Pareto fronts trading off various pairs of criteria are identified by means of a genetic algorithm. A different approach to a similar subject was investigated in [11]. In turn, in [12] the design optimisation of an electromagnetic valve actuator is proposed, and a suitable combination of three design criteria is exploited. In [13] the magnetic field in a permanent-magnet spherical motor at no-load is recovered, after inverting the magnetic induction measured along an accessible surface; the final aim is to compute the on-load torque by means of the Lorentz's law. In [14] an automated procedure of optimal shape design for MEMS was presented: it exploits finite-element analysis (FEA) for simulating the field in the device, and non-dominated genetic algorithm for trading off multiple objective functions.

The idea of using a multiobjective ranking system in biogeography optimisation was proposed e.g. in $[15,16]$; however, the explicit approximation of the Pareto front of non-dominated solutions is not presented. Moreover, the case studies considered are based on analytical models, the computation cost of which is low. In contrast, no case study characterized by numerical field models, which are challenging due to their high cost, is presented.

In the paper, a modification of a standard Biogeography-Based Optimisation (BBO) algorithm [17] in terms of the non-dominated sorting of habitats is proposed for design problems characterized by two conflicting criteria, the computation of which rely on field models. The modified algorithm Biogeography-inspired Multi-objective Optimisation (BiMO) was originnally introduced by the authors in [18] with reference to an electrostatic micromotor, which is a simple computational benchmark dating back to the origin of MEMS devices (see subsequent Section "2D numerical case study: optimal shape design of an electrostatic micromotor"). In [18] the authors discussed some technical features of the optimisation method like e.g. the effect of an error function driving the convergence, and the choice of the elitism operator in order to improve the approximation of the Pareto front.

The convergence of the proposed method was further investigated in [19], where the shape of the electrostatic micromotor was defined in a parameter-less way by means of the Bézier transform.

Here, based on the results presented in [18], the suitability of the proposed BiMO algorithm to help MEMS synthesis is investigated considering two case studies, i.e. the optimal shape design of two microactuators the operation of which exploits the electro-thermo-elastic interaction. In particular, a two-arms microactuator and a three-arms microactuator are considered, respectively. In the former case, the solution of the forward problem is based on an analytical model, while the analysis of the three-arms microactuator is based on a numerical field model.

The relevant Pareto fronts are explicitly identified for all devices; in particular, the field model of the three-arms microactuator implies the solution of a multi-physics analysis problem. 
A previous version of the proposed BiMO was applied to the shape synthesis of an electrostatic micromotor $[18,19]$; in this paper, a wider study on the applicability and suitability of the BiMO for solving shape design problems of MEMS is carried out. In the authors opinion, in fact, it is useful to investigate new optimisation methods for MEMS synthesis because, as the no free lunch theorem states [20], there are general purpose algorithms which perform on average well, but dedicated algorithms could perform better on a specific class of problems. The purpose of the paper is to investigate if the proposed BiMO exhibits a good performance when applied to this class of problems, i.e. the design optimisation of mechatronics devices.

The paper is organized as follows: the optimisation algorithm is presented, first; direct and inverse problems are defined for the microactuator problem, based on analytical model, and for the micromotor and microactuator problems, both based on numerical field models. Finally, relevant results are presented and discussed and a conclusion is drawn.

\section{Proposed optimisation method}

Computational biogeography models the process of natural immigration and emigration (or extinction) of species between small islands in the search for more friendly habitats, which is observed in nature [21].

\subsection{Migration and mutation}

Each solution considered in a biogeography-inspired optimisation algorithm (BBO) is treated as a habitat (design vector) composed of suitability index variables (SIV, design variables), and each habitat exhibits a quality given by the habitat suitability index (HSI, objective function). Remarkably, in contrast to GA-based algorithms, the original population is not discarded after each generation, but it is progressively modified by means of two stochastic operators, i.e. migration and mutation: migration improves the HSI of poor habitats by sharing features from good habitats; in turn, mutation modifies some randomly selected SIV of a few habitats in view of a better exploration of the ecosystem (design space). In practice, at each generation BBO exploits the HSI of each habitat based on its migration rate, while the emigration rate is set to be complementary to immigration. This way, the HSI of each habitat is improved (Fig. 1).

In particular, at each iteration, habitats are sorted from the best ones to the worst ones according to the relevant value of the generalized fitness value. For each SIV of each habitat a random event $\mathrm{r}_{j}$, such that $0<\mathrm{r}_{j}<\sup (\mathrm{I}, \mathrm{E})$ with I maximum immigration rate and $\mathrm{E}$ maximum emigration rate, is generated. Then value $r_{i}$ is compared with the corresponding immigration rate $\lambda_{\mathrm{i}}$ of the considered habitat. If $\mathrm{r}_{j}>\lambda_{i}$ then immigration occurs: the SIV considered in the current ecosystem migrates to the next ecosystem, keeping the same location (Fig. 2a).

In contrast, if $\mathrm{r}_{j}<\lambda_{i}$ emigration occurs (Fig. $2 \mathrm{~b}$ ): the current SIV in the considered habitat (say the $i$-th habitat) goes extinct and the SIV of another habitat (say the $k$-th habitat) takes the same SIV position in the $i$-th habitat of the next ecosystem. The $k$-th habitat is selected depending on the emigration rate and the SIV. 


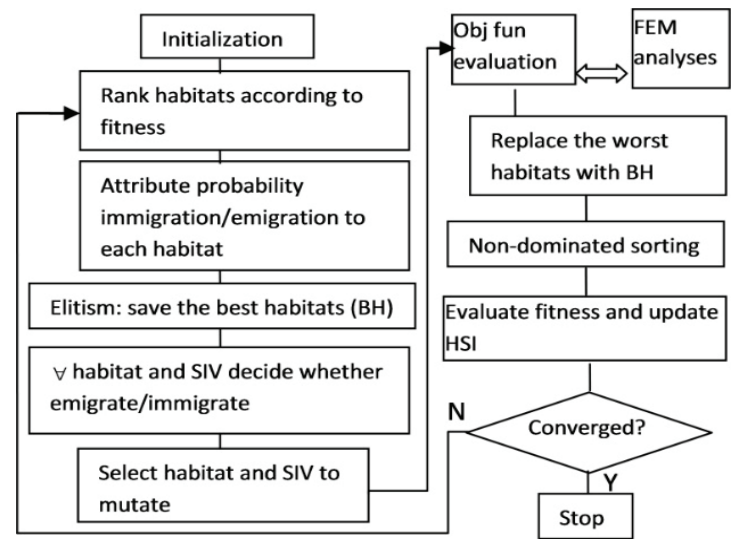

Fig. 1. Flow chart of the proposed BiMO algorithm

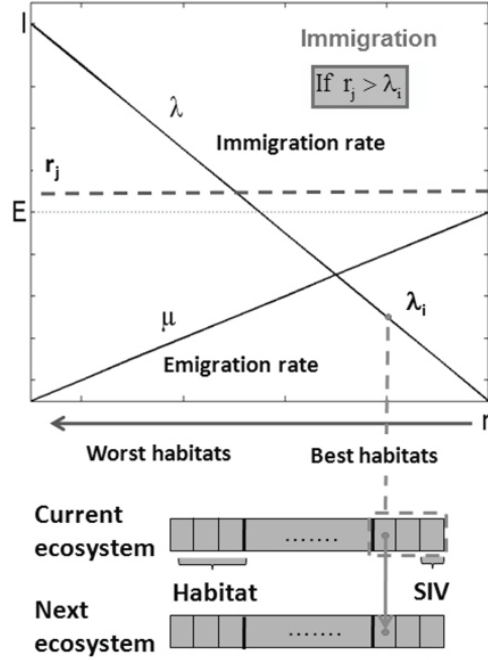

(a)

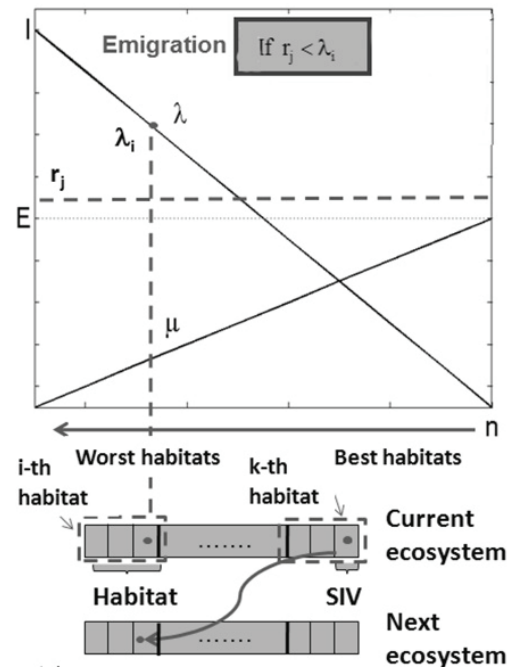

(b)

Fig. 2. Schemes of immigration and emigration events

\subsection{From single to multiple objective}

In computational electromagnetics, applications and also modifications of BBO are an emerging new field of research: various single-objective problems of optimal design have been successfully solved; moreover, attempts to transform BBO in a multi-objective optimisation algorithm have been considered [18, 19, 22-25]. In particular, the concept of predatorprey has been implemented [25] for hunting the worst individuals per objective function and making the surviving individuals spread in the objective space in order to escape from the predator. Though being interesting and effective, similar approaches might suffer from some limitations, like e.g. the possible loss of some non-dominated solutions (the worst individual per objective function might well be the end of the current set of non-dominated solutions), 
and the lack of an explicit criterion ruling the spread or crowd of the surviving individuals in the objective space.

As an alternative, it is proposed to modify the definition of HSI by means of a generalized fitness, which takes into account simultaneously two or more objective functions by exploiting the concept of non-dominated ranking of solutions in the objective space. At the current iteration, habitats are ordered according to two criteria: first, they are subdivided in locally nondominated sub-fronts which in turn are ranked from the first to the last. Next, habitats are ranked within the sub-front they belong to, taking into account their crowding distance; in fact, crowded habitats are less preferred than habitats which are regularly spaced.

At the end of a step-by-step procedure, a fitness value is assigned to each habitat; this makes it possible to sort the ecosystem from the best habitat to the worst one.

\subsection{Elitism operator}

Elitism is a technique for which the best solutions of the current iteration are passed, unchanged, to the next iteration. This is done for preserving the fittest solutions and hence for enhancing the exploitation capability of the method.

Hereinafter, it is assumed that the term "best" refers to the generalized fitness defined in this Section.

In [18] three different choices for the elitism operator were tested and applied in the electrostatic micromotor benchmark: two best habitats, a number of habitats equal to half of the Pareto front, a number of habitats equal to all solutions in the Pareto front, respectively. The recommended choice for a class of MEMS problems was proven to be the preservation of the best half of the Pareto front of the current ecosystem, which is then passed to the next one. In fact, the elitism exploiting the best half of the Pareto front is convenient to save runtime.

\subsection{Convergence criterion}

In order to rule the convergence of the proposed method, the following index is considered:

$$
m_{f}(k)=\frac{\left|d_{f}(k)-d_{f}(k-1)\right|}{d_{f}(k-1)},
$$

with

$$
d_{f}=\frac{1}{N_{p}} \sqrt{\sum_{i=1}^{N_{p}} \sum_{j=1}^{N_{f}}\left(\frac{f_{i, j}-u_{j}}{L_{j}}\right)^{2}},
$$

where: $N_{p}$ is the number of habitats, $N_{f}$ is the number of objective functions, $L_{j}$ is the distance between Utopia and Nadir points while $f_{i, j}$ and $u_{j}$ are the current values of the objective functions and the utopia point, respectively. Put simply, index $d_{f}$ is related to the mean distance of the current habitats to the Utopia point while index $m_{f}$ is calculated at the $k$-th iteration and gives information about how much the mean distance $d_{f}$ to Utopia changed at the $k$-th iteration with respect to the previous one. 


\section{Analytical case study: optimal shape design of a two-arms microactuator}

The laterally-driven polysilicon microactuator considered as a case study is the one studied in [26], where an analytical model of displacement and temperature is calculated. The microactuator is composed of two arms exhibiting different length; due to an applied voltage, a current flows in the series-connected arms. The current heats the device and because of the difference in length, the two heated arms elongates differently.

\subsection{Direct problem}

The deflection of the actuator depends on the following geometrical parameters: the length of the arms $L_{1}$ and $L_{2}$, the length $L_{g}$ of the gap between long and short arm, the height $h h$ of the device and the width $d$ of the arms (see Fig. 3).

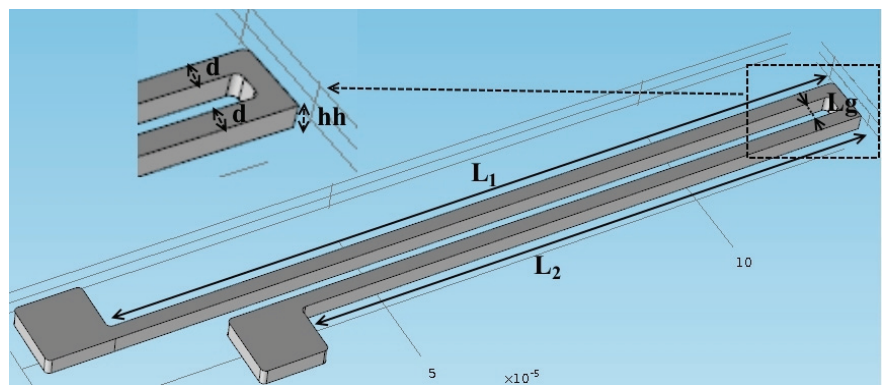

Fig. 3. Two-arms microactuator: the geometry and design variables are represented

In turn, the maximum temperature depends, from the geometrical viewpoint, on height $h h$ and width $d$. The dependencies on other parameters like e.g. the thermal conductivity are shown in [26]. The parameter values used in this paper are shown in Table 1.

Table 1. Material properties of the microactuator

\begin{tabular}{l|c|c|c}
\hline \multicolumn{1}{c|}{ Parameter } & Value & Parameter & Value \\
\hline Thickness of conducting layer $h c[\mu \mathrm{m}]$ & 0.5 & Thermal expansion coefficient $\alpha\left[{ }^{\circ} \mathrm{C}^{-1}\right]$ & $2.710^{-6}$ \\
\hline Thickness of top layer $h u[\mu \mathrm{m}]$ & hh-hc & Young modulus $[\mathrm{Pa}]$ & $15010^{9}$ \\
\hline Parameter $\xi$ & $1 / 9$ & Substrate temperature $T_{s}\left[{ }^{\circ} \mathrm{C}\right]$ & 20 \\
\hline Thermal conductivity $k\left[\mathrm{Wm}^{-1} \mathrm{~K}^{-1}\right]$ & 41 & Electrical resistivity $\rho[\Omega \mathrm{m}]$ & $510^{-4}$ \\
\hline
\end{tabular}

The parameter $\xi$ is used to take into account the difference of thermal conductivity and nonuniform heat generation between the top and the bottom layer [26]. The applied voltage is $5 \mathrm{~V}$.

\subsection{Inverse problem}

The optimisation of the geometry has been performed: the design variables are $L_{1}, L_{2}, L_{g}$, $h h$ and $d$. The objective functions are the maximum temperature $T_{m}$ to be minimized, and the displacement $U$, which is the deflection in the free end of the actuator, to be maximized. 
Two different algorithms have been applied: goal attainment method [20] and the proposed biogeography-based method.

Goal attainment is a well-known deterministic method, which allows to solve a multi-objective problem by reformulating it in terms of a minimax problem, subject to suitable weights expressing the user preferences. The minimax problem is subsequently solved by means of a eterministic algorithm, i.e. the sequential quadratic programming [27].

\section{2D numerical case study: optimal shape design of an electrostatic micromotor}

The electrostatic micromotor considered as the first case study exhibits 18 stator electrodes and 6 rotor teeth. It had been prototyped with the following data: inner and outer rotor radii 40 and $60 \mu \mathrm{m}$, stator radius $63 \mu \mathrm{m}$, angular width of rotor slot $40^{\circ}$ (Fig. 4). Accordingly, the width of the stator-to-rotor air-gap varies between 3 and $23 \mu \mathrm{m}$. The stator electrodes are supplied by a three-phase system of square voltages, equal to $100 \mathrm{~V}$, while the rotor potential is floating.

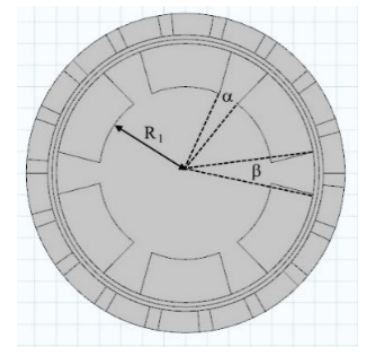

(a)

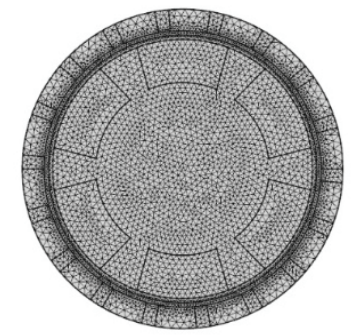

(b)

Fig. 4. Electrostatic micromotor: geometry (a) and finite-element mesh (b) about 9000 triangular elements and 18000 degrees of freedom

It is worth noting that electrostatic micromotors were the first MEMS which had been designed and prototyped exploiting Silicon integrated technology. This achievement took place at the Berkeley university laboratories first $[28,29]$, and at the MIT laboratories next $[30,31]$ in the period from late eighties through early nineties of last century. The cultural impact was great, because the miniaturization of a motor - well-known at the macro-scale - was implemented as a manufacturable process. However, the technological success of micromotors was modest, because static friction soon turned out to be the major obstacle against the rotor movement. Nevertheless, from the modeling viewpoint, the rotating micromotor is a good example of a simple device exhibiting the main features of MEMS, like e.g. mechanical and electrical properties of poly-silicon, size scale of components, and values of field strength. Therefore, it could be considered as a kind of computational benchmark useful to assess a numerical method for automated optimal design. 


\subsection{Direct problem}

The electric field analysis was based on a two-dimensional finite-element (FE) model of the micromotor based on the electric scalar potential. The field domain is the air-gap region; to account for friction, the side-pull effect has been considered, caused by the radial displacement of the rotor during its motion. Actually, the eccentric motion determines an unbalanced electric pull, which appears as a radial force acting in the direction of the shortest air-gap. Based on Maxwell's stress tensor method, the radial force acting on the rotor was computed as closed surface of integration embedding the rotor, and $\overline{\boldsymbol{n}}$ is the outward normal unit vector; specifically, the circle line passing along the air-gap centre was assumed as the integration line $\Gamma$. Moreover, to simulate the radial displacement, a clearance between rotor and shaft equal to $1 \mu \mathrm{m}$ was considered; the direction of displacement was assumed to be fixed and independent of $\phi$ (static eccentricity). In turn, the driving torque $M_{d}$ in no-load condition was computed as

$$
\bar{M}=\left(0,0, M_{d}\right)=\int_{\Gamma}^{-} \bar{r}_{P O} \times \overline{\bar{T}} \cdot \bar{n} d \Gamma,
$$

where: $\bar{r}_{P O}$ is the position vector of point $P$ on $\Gamma$ with respect to the rotation axis in $O$; it is assumed that rotor axis and stator axis are coincident. $M_{d}$ as a function of the angular position $\phi$ of the rotor is based on 120 points located along integration line $\Gamma$. The computational burden is substantial: for each angle position a new FE analysis is run, the Maxwell's stress tensor is updated and the relevant value of radial motive torque is computed.

\subsection{Inverse problem}

The shape design of the rotor has been considered, taking radius $R_{1}$ and angles $(\alpha, \beta)$ as the design variables. Significantly, two objective functions can be defined in terms of three-dimensional design vector $\boldsymbol{g}=\left(R_{1}, \alpha, \beta\right)$ namely:

- the highest value of driving torque on a pole pitch $f_{1}(g)$ at no load under single-phase supply;

- the value of radial force on the rotor $f_{2}(g)$ in the direction of the shortest air-gap.

The problem reads: given stator supply and rotor misalignment, find the family of rotor geometries $g$ such that $f_{1}(g)$ is maximum and $f_{2}(g)$ is minimum according to the Pareto definition of non-dominated solution. So, given a feasible geometry g according to the proposed optimisation algorithm, a FE analysis is run and vector objective function $f=\left[f_{1}(\boldsymbol{g})\right.$, $\left.f_{2}(g)\right]$ is updated.

\section{3D numerical case study: optimal shape design of a three-arms microactuator}

An electro-thermo-elastic microactuator is considered as the second case study, characterized by 3D geometric model and coupled field analysis. The device, shown in Fig. 5, has length $L$ in the range of hundreds of microns and width $h h$ in the range of tens of microns. An electric voltage is applied between two electrodes A and B; therefore, an electric current $I$ 
flows in two out of three arms of the device (hot arms), while the third arm is current free (cold arm).

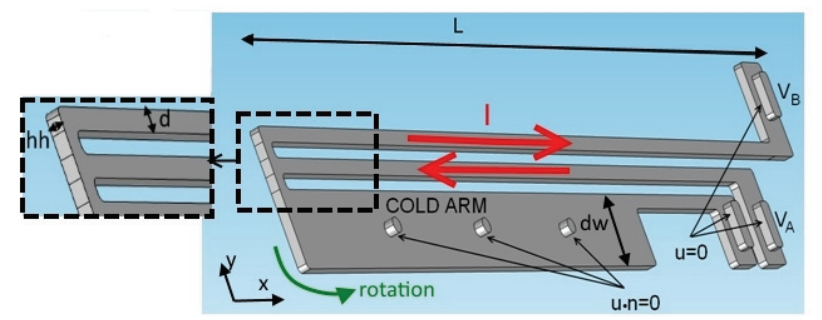

Fig. 5. Geometry of the microactuator, with the set $(L, h h, d w, d)$ of four design variables (electrical and mechanical boundary conditions are also shown)

The actuator is fixed to a substrate at the three arm ends, while three cylindrical bushings act on the cold arm, in order to make it rotate in the $x y$-plane (Fig. 5). The deformation due to the overheating of the hot arms with respect the cold one is responsible for the rotation of the actuator.

\subsection{Direct problem}

A 3D finite-element model of the device has been developed; a typical finite-element mesh is composed of about 8000 tetrahedra. The material properties used in the model are listed in Table 2.

Table 2. Material properties of the microactuator

\begin{tabular}{l|c|l|c}
\hline \multicolumn{1}{c|}{ Material property } & Value & \multicolumn{1}{c|}{ Material property } & Value \\
\hline Electric conductivity $\sigma\left[\mathrm{Sm}^{-1}\right]$ & $510^{4}$ & Young modulus $[\mathrm{Pa}]$ & $21010^{9}$ \\
\hline Thermal conductivity $k\left[\mathrm{Wm}^{-1} \mathrm{~K}^{-1}\right]$ & 34 & Fracture toughness $[\mathrm{Pa}]$ & $1.44-2.5110^{9}$ \\
\hline Thermal expansion coefficient $\alpha\left[\mathrm{K}^{-1}\right]$ & $2.610^{-6}$ & Poisson coefficient & 0.22 \\
\hline
\end{tabular}

The following multiphysics equations, which are coupled at the right-hand side level, are subsequently solved:

\section{Electric problem}

The governing equations are

$$
\nabla \cdot \sigma \nabla V=0
$$

and

$$
\boldsymbol{J}=\sigma \boldsymbol{E}=-\sigma \nabla V,
$$

subject to boundary conditions 


$$
V_{A}=\text { const, } V_{B}=0, \boldsymbol{n} \cdot \boldsymbol{J}=0 \text { elsewhere, }
$$

where: $\boldsymbol{J}$ is the current density, $\boldsymbol{E}$ the electric field and $V$ the unknown electric voltage.

\section{Thermal problem}

The governing equation is

$$
-\nabla \cdot(k \nabla T)=\boldsymbol{J} \cdot \boldsymbol{E},
$$

subject to boundary conditions

$$
\begin{gathered}
T=293.15 K \text { at the bushings and at the hinges, } \\
-\boldsymbol{n} \cdot(k \nabla T)=h\left(T_{\mathrm{ext}}-T\right) \text { elsewhere, }
\end{gathered}
$$

with: $\boldsymbol{J} \cdot \boldsymbol{E}$ the specific power due to the Joule effect, $T$ the unknown temperature and $h$ the convection coefficient; the latter has different values on the upper and the lower surface. In particular, the value of $h$ is equal to $400 \mathrm{Wm}^{-2} \mathrm{~K}^{-1}$ for the upper surface and $210^{4} \mathrm{Wm}^{-2} \mathrm{~K}^{-1}$ for the lower surface. These values are obtained by dividing the thermal conductivity of air $\left(0.04 \mathrm{Wm}^{-1} \mathrm{~K}^{-1}\right)$ by the distance to the surrounding surfaces for the system.

\section{Structural problem}

In order to solve the structural problem, the total strain $\varepsilon$ is additively decomposed into its elastic $\varepsilon^{e}$ and thermal $\varepsilon^{T}$ components

$$
\varepsilon=\varepsilon^{e}+\varepsilon^{T} .
$$

The thermal component, which reads

$$
\boldsymbol{\varepsilon}^{T}=\alpha\left(T-T_{\text {ref }}\right),
$$

is known after the cascade solution of (4) and (9).

This way, the classical continuum-mechanics problem, that incorporates compatibility, equilibrium and elastic equations, can be cast; they read

$$
\begin{gathered}
\boldsymbol{\varepsilon}=\frac{1}{2}\left(\nabla \boldsymbol{u}+\nabla \boldsymbol{u}^{T}+\nabla \boldsymbol{u} \nabla \boldsymbol{u}^{T}\right), \\
\nabla \cdot \boldsymbol{S}=0, \\
\boldsymbol{\varepsilon}^{e}=\boldsymbol{C}^{-1} \boldsymbol{S},
\end{gathered}
$$

respectively, where $u$ is the unknown displacement subject to

$$
u_{z}=0 \text { at the bushings, } u=0 \text { at the hinges, }
$$

while: $\boldsymbol{S}$ is the second Piola-Kirchhoff stress tensor, and $\boldsymbol{C}$ is the elastic constitutive tensor [32]. 
From the numerical viewpoint, the structural problem (12-15) is solved in the large displacement regime using the second Piola-Kirchhoff tensor as the stress measure, then the finite-element mesh is updated to simulate the deformed structure.

In Fig. 6 a solution of the multiphysics direct problem is shown; in particular, the field maps of temperature and stress are shown, respectively.

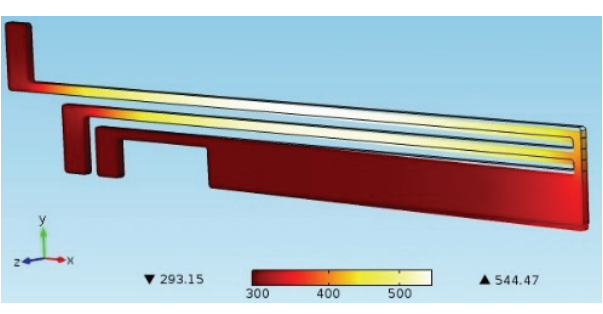

(a)

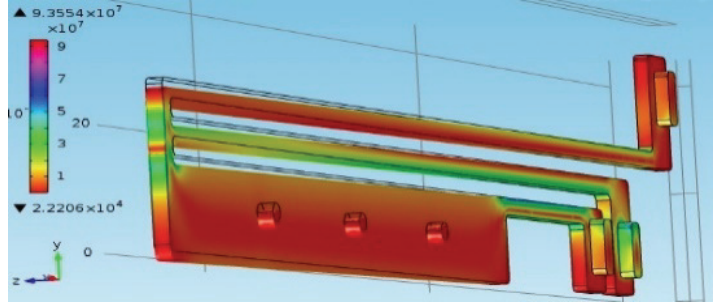

(b)

Fig. 6. Temperature (a) and stress (b) field maps

\subsection{Inverse problem}

The problem reads as follows: acting on the following design variables

$-L$ length of the actuator,

$-h h$ thickness of the actuator,

$-d w$ width of the cold arm,

$-d$ width of the hot arms,

two objective functions are defined:

$$
\begin{gathered}
f_{1}=\left.u\right|_{V=V \text { min }}+\left.u\right|_{V=V \text { max }}, \\
f_{2}=\left.T_{\max }\right|_{V=V \text { min }}+\left.T_{\max }\right|_{V=V \text { max }},
\end{gathered}
$$

where: $f_{1}$ is to be maximized and $f_{2}$ to be minimized, with $V_{\min }=1 \mathrm{~V}, V_{\max }=5 \mathrm{~V}$, subject to the constraints:

$$
\begin{gathered}
T_{\max }<1500 \mathrm{~K}, \\
\|S\|<1.44 \mathrm{GPa} .
\end{gathered}
$$

The meaning of the (19) is that the norm of the stress at the most solicited point of the actuator should not exceed the silicon breaking stress.

The design variable ranges are $L[56$ - 300] $\mu \mathrm{m}, h h[2-5] \mu \mathrm{m}, d w[7-30] \mu \mathrm{m}, d[1-7] \mu \mathrm{m}$, respectively.

For making the computation work, an automated procedure implementing the flow chart shown in Fig. 1 has been realized: it links the proposed algorithm BiMO to a standard code for FEA; the procedure was assessed by means of several numerical experiments. 


\section{Two-arms microactuator: optimisation results}

The BiMO algorithm has been applied with the following stopping criterion: the computation stops when mean $m_{f \text {,mean }}$ and maximum $m_{f, \text { max }}$ values of $m f$ over the last five iterations fulfill the following condition: $m_{f \text {,mean }}<1 \%$ and $m_{f \text {, max }}<2 \%$.

The starting points are randomly generated.

Goal attainment method has been used with the default settings for the routine goal attainment of MatLab [27]. The point $\left[-10^{-6}, 369\right]$ is taken as the goal vector, while the weights are the absolute value of the two component of the goal vector.

The optimisation results are shown in Fig. 7, where also a random sampling of the search space is shown (dots).

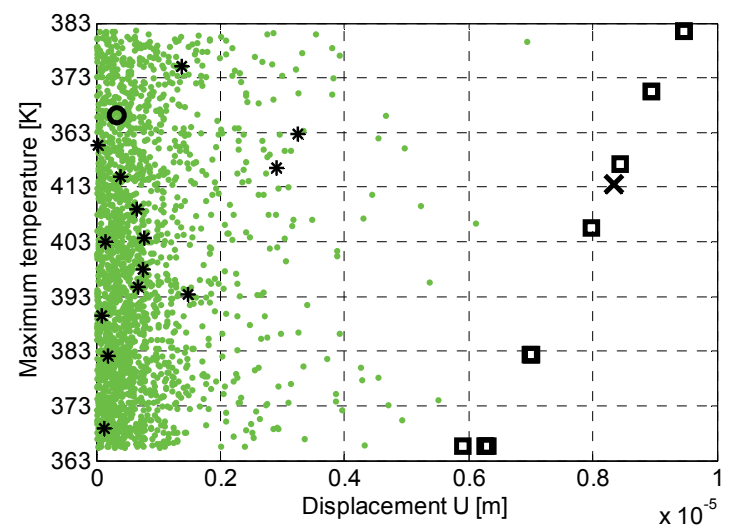

Fig. 7. Optimisation results: star - starting points BiMO, square - arrival points BiMO, circle - starting point goal attainment, cross - arrival point goal attainment, dot - random sampling.

The starting point for the goal attainment algorithm is the star while the arrival point is the cross. Goal attainment is able to find a solution belonging to the Pareto front. The run lasted 75 iterations (533 objective function calls).

BiMO algorithm is able to find a good approximation of the whole front. In fact, 13 points out of 14 converged to the approximated front. The run lasted 806 iterations (11 284 objective function calls).

In order to approximate the whole front with the goal attainment algorithm, it would be necessary to run many times the algorithm, each time varying its weights.

In order to make a comparison between the two algorithms, many runs should be done, and mean and standard deviation should be calculated. This is out of the scope of the paper. However, it is possible to say that both methods find Pareto-optimal solutions with hundreds of objective function calls per individual.

Moreover, the computational cost is comparable, if one considers that BiMO takes more time but finds 14 solutions, while goal attainment finds out just one solution. The computation time could be substantially high when considering numerical models for solving the forward problem. 
In order to make a comparison between the results found for the two-arms microactuator and the three-arms microactuator (Section "Three-arms microactuator: optimisation results"), it can be stated that the maximum value of temperatures for the two Pareto fronts are comparable. It can be noted from (17) that the objective function $\mathrm{f}_{2}$ is the sum of two temperatures; hence, the temperature values in Fig. 9 have to be approximately doubled in order to be compared with results in Fig. 9.

Also the displacements found for both devices exhibit the same order of magnitude.

\section{Micromotor: optimisation results}

Referring to Section "Proposed optimisation method", for a given choice of emigration and immigration rates, the mutation operator randomly modifies a SIV for each habitat depending on the mutation probability. The ultimate goal is to keep a good balance between exploration of design space (mutation helped) and exploitation of some promising habitats (migration rate assisted). In particular, for BiMO method, the mutation probability is set to 0.04 , based on a heuristic approach.

In [19] it was found that for the class of inverse problems for MEMS synthesis, the nonlinearity of the immigration and emigration curves does not affect substantially the solution: therefore, a linear model of immigration-emigration curve with $E=I=1$ is here considered (see Fig. 2).

In Fig. 8 the approximated Pareto front found by means of BiMO method is shown; for the sake of a comparison, the front obtained by means of NSGA-II is also shown (both methods stopped after $n_{g}=5$ generations, each based on $n_{p}=14$ individuals). For each method the overall cost is proportional to $n_{p} \times n_{g}$ calls to the FEA. Each call to FEA lasts about 1-2 minutes on a computer equipped with a processor Intel i7, $3.6 \mathrm{GHz}$ and $16 \mathrm{~GB}$ of RAM.

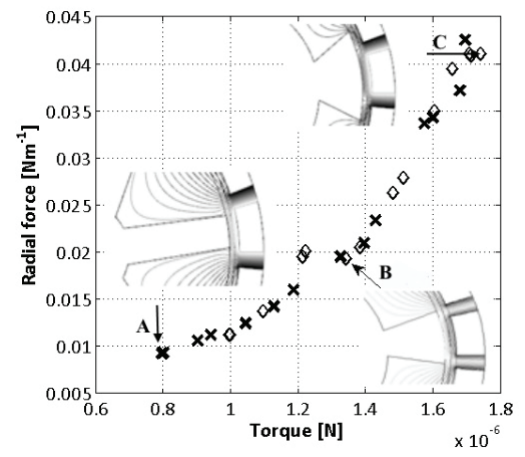

Fig. 8. Objective space and non-dominated solutions: cross - NSGA-II results, diamond - BiMO multi-objective results

In Fig. 8 geometry and potential lines of non-dominated solutions A, B and C are also shown. 
These solutions are characterized by design variable values and objective function values shown in Table 3.

Table 3. Results of A, B and C solutions in Fig. 8

\begin{tabular}{l|c|c|c|c|c}
\hline & $\boldsymbol{R}_{\mathbf{1}}[\boldsymbol{\mu} \mathbf{m}]$ & $\boldsymbol{\alpha}[\mathbf{d e g}]$ & $\boldsymbol{\beta}[\mathbf{d e g}]$ & Torque $[\boldsymbol{\mu} \mathbf{N}]$ & Radial force $\left[\mathbf{m N m}^{-1}\right]$ \\
\hline Sol. A & 18.6 & 83.4 & 10.0 & 0.80 & 9.30 \\
\hline Sol. B & 39.8 & 11.6 & 23.1 & 1.34 & 19.4 \\
\hline Sol. C & 41.2 & 11.6 & 40.8 & 1.74 & 41.1 \\
\hline
\end{tabular}

\section{Three-arms microactuator: optimisation results}

Considering the good performance of the elitism technique applied in the study of the [18], half of the Pareto front is considered for elitism. In fact, using half of the Pareto front for elitism is suitable when a time-consuming forward problem (like (4)-(15) is in 3D) has to be solved at each iteration.

An ecosystem composed of $n_{i}=14$ islands is considered; the stopping criterion is based on a twofold condition as follows: the optimisation stops when the mean value of $m_{f}$ considering the last 5 iterations, $m_{f \text {,mean }}$, is smaller than a prescribed threshold, e.g. 0.01 and the maximum value of $\mathrm{mf}$ considering the last 5 iterations, $m_{f, \max }$, is smaller than a prescribed threshold, e.g. 0.02 .

The optimisation lasted $n_{g}=35$ iterations. At each iteration the Pareto front is calculated and archived, considering also the history of the optimisation results: the Pareto front so found is shown in Fig. 9. For each method the overall cost is proportional to $n_{i} \times n_{g}$ calls to the FEA. Each call to FEA lasts, depending on the mesh discretizing the geometry, from 5 through 20 minutes on a computer equipped with a processor Intel i7, $3.6 \mathrm{GHz}$ and $16 \mathrm{~GB}$ of RAM.

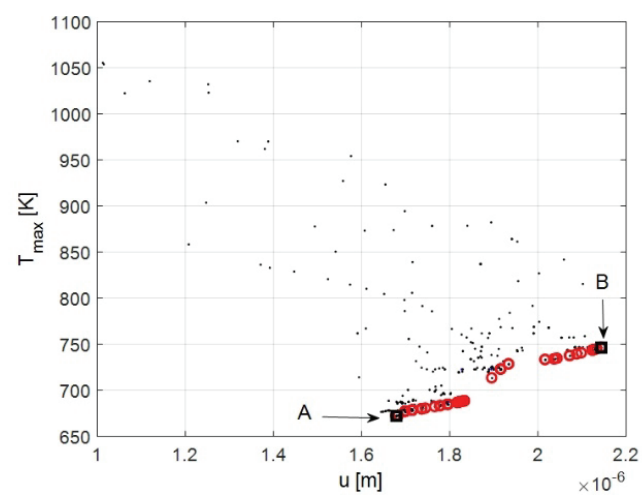

Fig. 9. Objective space (dot) and non-dominated solutions (circle); A and B points (square) are the ends of the approximated Pareto front 
The temperature maps of solutions A and B (Fig. 9) are shown in Fig. 10.

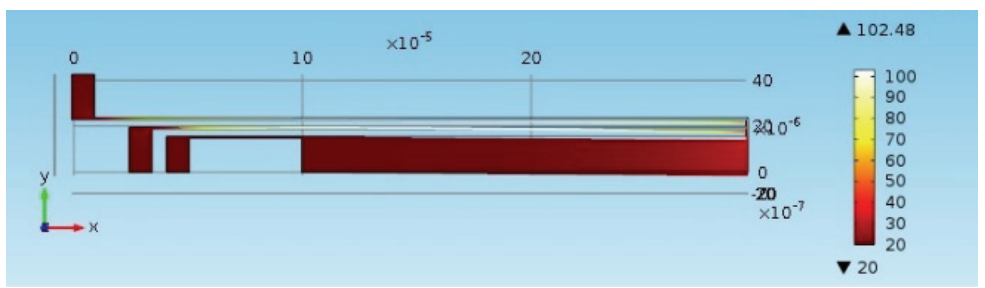

(a)

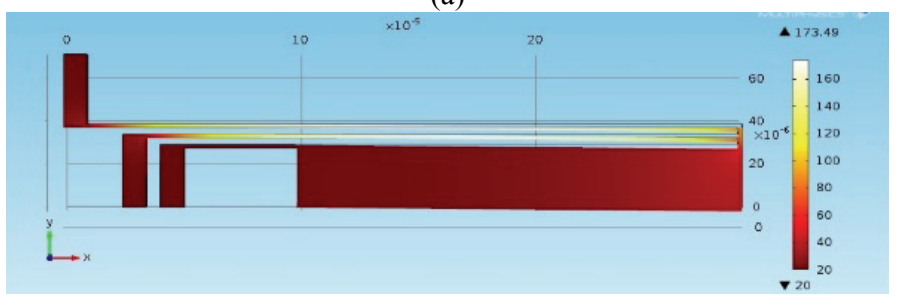

(b)

Fig. 10. Temperature $\left[{ }^{\circ} \mathrm{C}\right]$ maps of solutions A (a) and B (b)

Two solutions, located at the end points of the Pareto front, are considered. Results in terms of design variable, objective function and constraint values are shown in Table 4.

Table 4. Results of the two end-points of the Pareto front in Fig. 9

\begin{tabular}{c|c|c|c|c|c}
\hline $\begin{array}{c}\text { Design variables } \\
{[\boldsymbol{\mu} \mathbf{m}]}\end{array}$ & A & B & $\begin{array}{c}\text { Objective function/ } \\
\text { constraint }\end{array}$ & A & B \\
\hline$L$ & 293.75 & 286.9 & $f_{1}[\mu \mathrm{m}]$ & 1.68 & 2.14 \\
\hline$h h$ & 2.03 & 4.97 & $f_{2}[\mathrm{~K}]$ & 672.2 & 746.3 \\
\hline$d w$ & 15.76 & 29.13 & $T_{\max }[\mathrm{K}]$ & 375.6 & 446.6 \\
\hline$d$ & 1.00 & 1.72 & $S[\mathrm{GPa}]$ & 0.19 & 0.44 \\
\hline
\end{tabular}

Taking into account the results in Table 4, it can be stated that, from the designer point of view, a long actuator should be preferred. Moreover, considering the bounds of the height $h h$, it can be observed that the value of $h h$ is the smallest one for device A and the highest one for device B. A further role is played by the ratio $d w$ to $d$, which should be high. Therefore, according to extra preferences of the device designer, a final choice can be made a posteriori among Pareto-optimal solutions.

In order to compare this method with a well-known classical method, the goal attainment algorithm (the same used for the two-arms microactuator, Section "Two-arms microactuator: optimisation results") has been resorted to. However, after many numerical experiments, it was not able to find any non-dominated solution on the Pareto front. This is probably due to the lack of differentiability of objective functions (16)-(17), which makes the problem stiff for a deterministic method like goal attainment. 


\section{Conclusion}

The proposed method extends a standard bio-geography based optimisation algorithm to the multi-objective case by modifying the definition of fitness. The optimal shape designs of a two-arms microactuator and of an electrostatic micromotor have been considered as case studies for studying the features of the proposed method; in turn, a more realistic application, the microactuator, has been optimized exploiting the results obtained with the first case studies. To the best knowledge of the authors, in the area of methods for MEMS design optimisation, biogeography-inspired optimisation appears to be a new and promising design tool.

\section{References}

[1] Di Barba P., Dolezel I., Karban P., Kus P., Mach F., Mognaschi M.E., Savini A., Multiphysics field analysis and multiobjective design optimization: a benchmark problem, Inverse Problems in Science and Engineering (IPSE), vol. 22, no. 7 (2014).

[2] Di Barba P., Mognaschi M.E., Sorting Pareto solutions: a principle of optimal design for electrical machines, The International Journal for Computation and Mathematics in Electrical and Electronic Engineering - Compel, vol. 28 no. 5, pp. 1227-1235 (2009).

[3] Sieni E., Di Barba P., Forzan M., Migration NSGA: method to improve a non-elitist searching of Pareto front, with application in magnetics, Inverse Problems in Science and Engineering (IPSE), vol. 4, pp. 543-566 (2016).

[4] Deb K., Multi-Objective Optimisation Using Evolutionary Algorithms, Wiley (2001).

[5] Coelho L.S., Alotto P., Electromagnetic Optimization Using a Cultural Self-Organizing Migrating Algorithm Approach Based on Normative Knowledge, IEEE Trans. Magn., vol. 45, no. 3, pp. 1446-1449 (2009).

[6] Deep K., A self-organizing migrating genetic algorithm for constrained optimization, Appl. Math. Comput., vol. 198, no. 1, pp. 237-250 (2008).

[7] Tang W., Nguyen T. and Howe R., Laterally driven polysilicon resonant microstructures, Sensors and actuators, vol. 20, pp. 25-32 (1989).

[8] Boudaoud M., Haddab Y., Le Gorrec Y., Modeling and optimal force control of a nonlinear electrostatic microgripper, IEEE/ASME Trans. Mechatronics, vol. 18, no. 3, pp. 1130-1139 (2013).

[9] Delinchant B., Rakotoarison H.L., Ardon V., Chabedec O., Cugat O., Gradient based optimization of semi-numerical models with symbolic sensitivity: Application to simple ferromagnetic MEMS switch device, IJAEM, vol. 30, pp. 189-200 (2009).

[10] Grossard M., Rotinat-Libersa M.C., Chaillet N., Boukallel L., Mechanical and control-oriented design of a monolithic piezoelectric microgripper using a new topological optimization method, IEEE/ASME Trans. Mechatronics, vol. 14, no. 1, pp. 32-45 (2009).

[11] Di Barba P., Lorenzi A., A Magneto-thermo-elastic identification problem with a moving boundary in a micro-device, Milan J. of Mathematics, vol. 81, pp. 347-383 (2013).

[12] Yang Y.P., Liu J.J., Ye D.H., Chen, Y.R., Lu P.H., Multiobjective Optimal Design and Soft Landing Control of an Electromagnetic Valve Actuator for a Camless Engine, IEEE/ASME Trans. Mechatronics, vol. 18, no. 3, pp. 963-972 (2013).

[13] $\mathrm{Hu}$ L., Lee K.M., Fu X., A method based on measured boundary conditions for reconstructing the magnetic field distribution of an electromagnetic mechatronic system, IEEE/ASME Trans. Mechatronics, vol. 15, no. 4, pp. 595-602 (2010).

[14] Di Barba P., Wiak S., Evolutionary Computing and Optimal Design of MEMS, IEEE Trans. on Mechatronics, vol. 20, no. 4, pp. 1660-1667 (2015).

[15] Du D., Simon D., Complex system optimization using biogeography-based optimization, Mathematical Problems in Engineering (2013). 
[16] Ma H., Su S., Simon D., Fei M., Ensemble multi-objective biogeography-based optimization with application to automated warehouse scheduling, Engineering Applications of Artificial Intelligence, vol. 44, pp. 79-90 (2015).

[17] Simon D., Biogeography-Based Optimization, IEEE Trans. on Evol. Comput., vol. 12, no. 6, pp. $702-713$ (2008).

[18] Di Barba P., Dughiero F., Mognaschi M.E., Savini A., Wiak S., Biogeography-Inspired Multiobjective Optimization and MEMS Design, IEEE Trans. on Magnetics, vol. 53, no. 3 (2016).

[19] Di Barba P., Mognaschi M.E., Savini A., Wiak S., Island Biogeography as a Paradigm for MEMS Optimal Design, International Journal of Applied Electromagnetics and Mechanics IJAEM, vol. 51(s1), pp. 97-105 (2016).

[20] Di Barba P., Multiobjective Shape Design in Electricity and Magnetism, Springer (2010).

[21] Macarthur R.H., Wilson E.O., The Theory of Island Biogeography, Princeton University Press (1967).

[22] Roy P.K., Ghoshal S.P., Thakur S., Multi-objective Optimal Power Flow Using Biogeographybased Optimization, Electric Power Components and Systems, vol. 38, pp. 1406-1426 (2010).

[23] Singh S., Mittal E., Sachdeva G., NSBBO for Gain-Impedance Optimization of Yagi-Uda Antenna Design, Proc. 2012 World Congress on Information and Communication Technologies, pp. 856-860 (2012).

[24] Singh U., Kumar H., Kamal T.S., Design of Yagi-Uda Antenna Using Biogeography Based Optimization, IEEE Trans. on Antennas and Propagation, vol. 58, no. 10, pp. 3375-3379 (2010).

[25] Costa Silva M.A., Coelho L.S., Lebensztajn L., Multiobjective Biogeography-Based Optimization based on Predator-Prey Approach, IEEE Trans. on Magnetics, vol. 48, no. 2, pp. 951-954 (2012).

[26] Huang Q.A., Lee N.K.S., Analytical modeling and optimization for a laterally-driven polysilicon thermal actuator, Microsystem technologies, vol. 5, pp. 133-137 (1999).

[27] http://www.mathworks.com, last visited on 18th October 2016.

[28] Fan L.S., Tai Y.C., Muller R., IC processed electrostatic microactuators, Sensors and Actuators, vol. 20, pp. 41-47 (1989)

[29] Fan L.S., Tai Y.C., Muller R., IC processed electrostatic synchronous microactuators, Sensors and Actuators, vol. 20, pp. 49-55 (1989).

[30] Mehregany M., Senturia S.D., Lang J.H., Nagarkar P., Micromotor fabrication, IEEE Trans. Electron Devices, vol. 39, pp. 2060-2069 (1992).

[31] Bart S.F., Mehregany M., Tavrow L.S., Lang J.H., Senturia S.D., Electric Micromotor Dynamics, IEEE Trans. on Electron Devices, vol. 39, no. 3 (1992).

[32] Bonet J., Wood R.D., Nonlinear continuum mechanics for finite element analysis, Cambridge University Press (2008). 